\title{
Mobile ECG Measurement and Analysis System Using Mobile Phone as the Base Station
}

\author{
Harri Kailanto, Esko Hyvärinen and Jari Hyttinen \\ Ragnar Granit Institute \\ Tampere University of Technology \\ Tampere, Finland \\ harri.kailanto@tut.fi
}

\begin{abstract}
In this paper, we introduce an ECG measurement, analysis and transmission system which uses a mobile phone as a base station. The system is based on a small-sized mobile ECG recording device which sends measurement data wirelessly to the mobile phone. In the mobile phone the received data is analyzed and if abnormalities are found part of the measurement data is sent to a server for the use of medical personnel. The prototype of the system was made with a portable ECG monitor and Nokia 6681 mobile phone. The results show that modern smart phones are indeed capable for this kind of tasks. Thus, with their good networking and data processing capabilities, they are a potential part of the future wireless health care systems.
\end{abstract}

Keywords; mobile health care; ECG; wireless; Bluetooth; $J 2 M E$

\section{INTRODUCTION}

Smart Home in a Suitcase - Service Platform for Assisted Living and Safety (UUTE) project is organized in co-operation between four institutes of Tampere University of Technology and VTT Technical Research Centre of Finland [1]. The aim of the project is to develop a so called "Smart home in a suitcase" concept, and the special emphasis is put on providing support for selected groups of patients needing support to manage their disease or improve the rehabilitation.

As a part of UUTE project, we developed a new kind of mobile ECG measurement and analysis system using the mobile phone as a base station. The system was planned to be used for example in the adjustment of the medication of cardiac arrhythmia patients. The main idea was that, to lower the work load of the medical personnel and to speed up the measurement to analysis cycle, the analysis should mainly be done automatically near the person under measurement (patient) and only in critical cases data should be sent to medical personnel for making a more accurate diagnosis. Another main concern was that mobility of the patient should not be limited more than absolutely necessary.

There already exist mobile measurement systems which use mobile phones or PDAs as gateways to send measurement data continuously to a separate server where the actual analysis is done, such as the one proposed by Chen it al. [2]. This kind of continuous sending may however be costly both financially, as data communication costs on mobile networks are often related to the sent amount of data, and in the sense of battery life [3].

Using short range radio technologies, such as Bluetooth, is free of charge and less power consuming than using long range technologies, so this kind of radio can be used to send the data straight to the $\mathrm{PC}$ of the patient with out financial costs. On the PC the analysis can be done or the data can be sent forward to a separate server. This kind of systems already exist as well, such as @ Home system [4].The problem with the systems using PC as a base station is, however, that they limit the moving range of the patient for he should stay close to the PC and cannot leave for example his apartment.

The computing power of modern smart phones has increased a lot so we decided to develop a system where automated analysis is done already in the phone which can be expected to stay close enough to the measured person to use short range radios. Only the parts of the data where the analysis application would find abnormalities were to be sent to the server for further analysis.

Similar kind of system has been proposed at least by Rodríquez et al. [3] with the difference that they are using PDA, not smart phone, as a base station. Leijdekkers \& Gay [5] have also developed a system where smart phone is used to analyze the ECG. Their system, however, does not send the actual measurement data along with the alarms.

\section{SYSTEM DESCRIPTION}

The base of the system consists of the measurement device described in the following and Nokia 6681 smart phone. The measurement device measures two channels of ECG and acceleration in two directions. It converts the analogue signals into digital form and sends them into the phone using Bluetooth connection.

The phone receives the transmitted data and saves it to a memory card for the later usage by medical personnel. It also analyzes the ECG signal and in the case of abnormalities, sends part of the measurement data to a server for further analysis by e.g. medical personnel. When requested, the application also displays the ECG signal and heart rate on the phone screen. The main tasks of the different parts of the system are presented in Figure 1.

\section{A. Measurement Device}

The measurement device used for the ECG measurements is based on the earlier design by Hyvärinen et al. [6]. The difference to the earlier design is that data logger module is replaced with a Bluetooth transceiver.

Two channels of ECG are measured. The sampling frequency for the ECG signals is $200 \mathrm{~Hz}$ and the voltage values are coded 


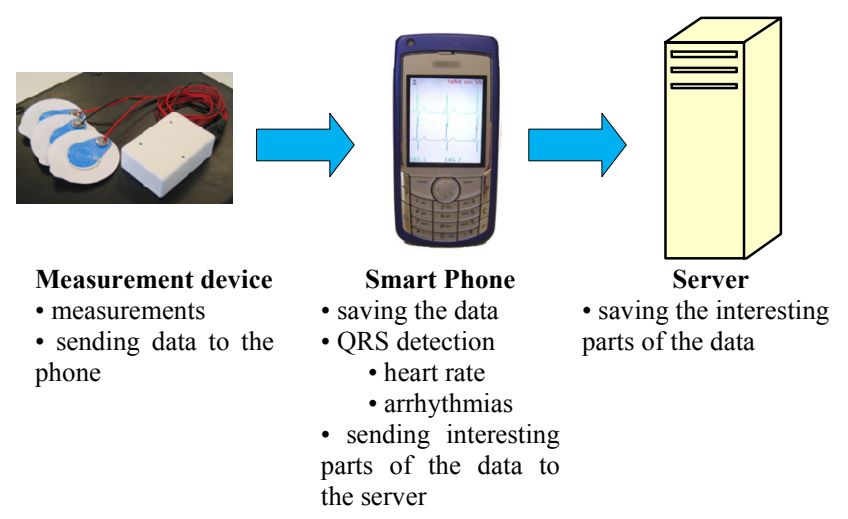

Figure 1. Main tasks of the different parts of the system.

with 8 bits. The achieved voltage resolution is about $0.023 \mathrm{mV}$ and the measurement range is $\pm 3 \mathrm{mV}$. In addition, the device measures 2-dimensional acceleration signal but these signals are not used in the described system at the moment.

The measured signals are first filtered with a bandpass-filter (highpass limit $0.07 \mathrm{~Hz}$ and lowpass limit $86 \mathrm{~Hz}$ ) and amplified with gain 500 to a reasonable level for further processing. After the filtering and amplification, signals are digitalized and packed into a byte array of 512 bytes. Each package includes the measured data from the window of $1120 \mathrm{~ms}$. After the packaging is done, data package with these 512 bytes is sent via Bluetooth Serial Port Profile to the smart phone using 115.2 kbps data rate. Thus, transmission time for one package is 40 ms.

\section{B. Application Program in the Phone}

The application on the smart phone is programmed on Java Platform, Micro Edition. It uses CLDC 1.1 and MIDP 2.0 with Bluetooth API, WirelessMessaging API and FileConnection API.

The application receives the data sent by the measurement device, saves it to a file in the phone and separates the two channels of the ECG signal for the analysis. In addition, the received data is saved to a separate buffer to keep the last 56 seconds of the measurement data stored for the possible transmission to a health care server. The reason of using different buffer for this task is that reading from the end of the file where all the data is saved would be time consuming when the file gets big enough.

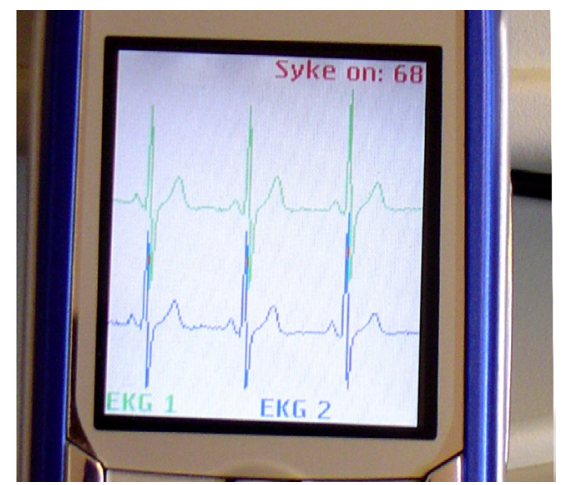

Figure 2. The phone screen when the signal drawing mode is activated.
From every received ECG data set, the application finds the QRS complexes using a simple derivative based algorithm. Based on the found QRS complexes it calculates the heart rate of the patient. If the heart rate is below or above of the pre-set limits, the last 56 seconds of measurement data preceding this cardiac event (from the buffer) along with the 56 seconds of data following the event is transmitted via Hypertext Transfer Protocol (HTTP) -connection to a separate server for additional analysis e.g. by medical personnel.

In addition to automated analysis, the application offers a possibility for a patient to send an alarm if he feels anxiety, faintness or other distress. The result of sending an alarm is the same as exceeding the heart rate limits. That is, a predefined amount of measurement data around this event is sent to the server. In addition, an informative Short Message Service (SMS) -message is delivered to the mobile phone of a selected person if so chosen.

There is also a possibility to view the received ECG signals on the phone screen along with the measured heart rate, as illustrated in Figure 2.

\section{PERFORMANCE OF THE PROTOTYPE}

The purpose of the first tests was to test the general functionality of the system, not the used algorithms or accuracy of the analysis made in the phone. The distance between the measurement device and the mobile phone during the tests was kept less than five meters without major blocking objects between the devices. Otherwise, the subject was living his normal daily life during the testing.

The average battery life for the phone when the application was continuously running was about 16 hours which might cause a problem if long time monitoring is needed. However, this time should be sufficient if the person under measurement spends most of the time in a home environment where the phone can be attached to the mains power source.

A new data set from the measurement device is sent every 1.12 seconds. This is also the time that the application on the phone can use for data saving and analysis, for longer times would eventually lead to enormous delay between data receiving and analysis. The tests showed that with our current version of the application program the average time used for saving data was about $350 \mathrm{~ms}$, the time for data analysis about $30 \mathrm{~ms}$, and the time for drawing the signals on the screen about $40 \mathrm{~ms}$. When an alarm was raised and data was prepared to be sent to a server the time taken by the data saving occasionally exceeded three seconds which caused problems in form of data loss.

\section{DISCUSSION AND CONCLUSIONS}

We have developed a mobile ECG measurement and analysis system using a mobile phone as a base station. In this system, the computing capabilities of the phone are used to make signal processing and analysis of the measured signals. When abnormalities are found, part of the signal is sent to a server for further analysis by medical personnel.

The tests with the first prototype showed that the modern smart phones are very well capable for nearly real time signal analysis combined with data transmission. The application program time consumption analysis showed that most of the 
time was consumed by data saving whereas the actual analysis was promisingly fast. This suggests that a lot more accurate analysis could be performed with more complicated algorithms than in the current prototype.

The first prototype of the application program had problems with data handling in some situations which lead to various amounts of data loss. The problems are now thought to be solved through modifying the data handling methods. This also seems to have improved the overall time consumption of the application program.

\section{REFERENCES}

[1] UUTE, http://www.rgi.tut.fi/uute [last accessed 30th November 2007].
[2] Wenxi Chen; Wei, D.; Xin Zhu; Uchida, M.; Ding, S.; Cohen, M., "A mobile phone-based wearable vital signs monitoring system," Computer and Information Technology, 2005. The Fifth International Conference on, pp. 950-955, 21-23 Sept. 2005

[3] J. Rodriguez, L. Dranca, A. Goñi, A. Illarramendi, "Web access to data in a mobile ECG monitoring system," Stud Health Technol Inform. 2004;vol. 105, pp. 100-11.

[4] I. Sachapazidis, “@Home: A modular telemedicine system," Proc. $2^{\text {nd }}$ Workshop Mobile Computing in Medicine, Heidelberg, Germany, 2002.

[5] P. Leijdekkers, and V. Gay, "Personal Heart Monitoring System Using Smart Phones To Detect Life Threatening Arrhythmias," ComputerBased Medical Systems, 2006. 19th IEEE International Symposium on, pp. 157-164.

[6] E. Hyvärinen, I. Ruuskanen, J. Hyttinen, J. Kaihilahti, O. Vainio, "Mental Stress Analysis Instrumentation and Tools Based On Heart Rate Variability" Analysis Of Biomedical Signals And Images 18th International EURASIP Conference Biosignal 2006 Proceedings; Brno VUTIUM Press 2006, pp. 196-198. 